\title{
छூ \\ Highly coherent vacuum ultraviolet radiation at the 15 th harmonic with echo-enabled harmonic generation technique
}

\author{
E. Hemsing, M. Dunning, C. Hast, T. O. Raubenheimer, S. Weathersby, and D. Xiang ${ }^{*}$ \\ SLAC National Accelerator Laboratory, Menlo Park, California 94025, USA
}

(Received 3 February 2014; published 21 July 2014)

\begin{abstract}
$\mathrm{X}$-ray free-electron lasers are enabling access to new science by producing ultrafast and intense $\mathrm{x}$ rays that give researchers unparalleled power and precision in examining the fundamental nature of matter. In the quest for fully coherent $\mathrm{x}$ rays, the echo-enabled harmonic generation technique is one of the most promising methods. In this technique, coherent radiation at the high harmonic frequencies of two seed lasers is generated from the recoherence of electron beam phase space memory. Here we report on the generation of highly coherent and stable vacuum ultraviolet radiation at the 15th harmonic of an infrared seed laser with this technique. The experiment demonstrates two distinct advantages that are intrinsic to the highly nonlinear phase space gymnastics of echo-enabled harmonic generation in a new regime, i.e., high frequency up-conversion efficiency and insensitivity to electron beam phase space imperfections. Our results allow comparison and confirmation of predictive models and scaling laws, and mark a significant step towards fully coherent $\mathrm{x}$-ray free-electron lasers that will open new scientific research.
\end{abstract}

DOI: 10.1103/PhysRevSTAB.17.070702

PACS numbers: 41.60.Cr

\section{INTRODUCTION}

Free-electron lasers (FELs), in which intense radiation with continually tunable wavelength is produced from relativistic electrons wiggling through a long magnetic undulator, are enabling new sciences in various areas, such as nanocrystallography and single molecule imaging [1-3], in situ studies of micrometer-size particulate matter [4], stimulated x-ray Raman scattering [5], femtosecond switching in electronics [6], and x-ray optical wave mixing [7], just to name a few. Currently, most of the x-ray FELs [8-10] operate in the self-amplified spontaneous emission (SASE) mode $[11,12]$ in which the initial beam shot noise seeds the FEL process and is exponentially amplified to the gigawatt $(\mathrm{GW})$ level through resonant interaction between the electron beam and the radiation field. While the excellent transverse coherence in SASE FELs enables numerous applications in coherent diffraction imaging, the random shot noise that seeds the amplification process leads to poor temporal coherence (e.g., noisy in both temporal profile and spectrum), which limits applications in resonant scattering and spectroscopic techniques.

One natural way to improve the temporal coherence of SASE FELs is to initiate the FEL process with a fully coherent seed of sufficient power to dominate over the

\footnotetext{
"Present address: Shanghai Jiao Tong University, Shanghai 200240, China.

dxiang@sjtu.edu.cn

Published by the American Physical Society under the terms of the Creative Commons Attribution 3.0 License. Further distribution of this work must maintain attribution to the author $(s)$ and the published article's title, journal citation, and DOI.
}

electron beam shot noise, thereby overcoming the SASE startup process. In the self-seeding technique [13-15] such a seed is from the SASE FEL itself, i.e., a monochromator is used to purify the spectrum of a SASE FEL and an additional undulator is employed to amplify the purified radiation to GW level. However, the intrinsically noisy properties of the SASE radiation in the first undulator lead to large intensity fluctuations in the monochromatized radiation which may affect the stability of the final output. In direct seeding with a high harmonic generation (HHG) source (see, for example [16]), a high power laser is injected into a noble gas to produce fully coherent radiation at a high harmonic of the laser, which is further amplified by several orders of magnitude by the FEL instability process. Limited by the $\sim 10^{-6}$ conversion efficiency, the shortest HHG wavelengths that can be used are around $\sim 20 \mathrm{~nm}$ due to the relatively low power $(\sim 100 \mathrm{~kW})$. While there is progress from the laser community in extending the HHG wavelength to $5-10 \mathrm{~nm}$, and from the accelerator community in extending direct seeding to $160 \mathrm{~nm}$ [17], $61 \mathrm{~nm}$ [18] and more recently to $38 \mathrm{~nm}$ [19], seeding with HHG sources in the x-ray wavelength still requires major advancement in laser technology because the HHG power needs to be at least 2 orders of magnitude larger than the electron beam shot noise power (increases as wavelength decreases) in order to preserve the temporal coherence.

To circumvent the need for a high power seed at short wavelengths, many frequency up-conversion techniques that rely on laser manipulation of the electron beam phase space have been proposed [20-28]. In these schemes, coherent charge density modulations, or "bunching," significantly above the shot noise is generated in the electron beam to stimulate emission of coherent radiation in the 
beginning of the FEL that is amplified to saturation. The leading techniques can be classified as either high-gain harmonic generation (HGHG) or echo-enabled harmonic generation (EEHG) and their variants. All of the techniques rely on first producing an energy modulation with lasers in the relativistic electron beam phase space. Then, the beam is sent through dispersive elements (typically a four-dipole chicane) that allows electrons with different energies to follow different paths. The result is a longitudinal rearrangement that yields a charge density modulation at wavelengths much shorter than the modulating laser (see, for example, [29]).

The HGHG technique uses one modulator-chicane system to produce density modulation at the high harmonics of the seed laser. While simple, this scheme is characterized by limited frequency up-conversion efficiency because generation of the $h$ th harmonic bunching typically requires the energy modulation to also be $\sim h$ times larger than the initial beam energy spread. Because large increases in the beam energy spread deteriorate the beam quality and significantly reduce the FEL gain, the harmonic number practically achievable is typically limited to about 10 and below [30,31]. In order to generate coherent soft $\mathrm{x}$ rays with wavelengths of a few nanometers from an ultraviolet (UV) seeding laser, multiple stages of the HGHG FEL are used [32,33]. While effective, the performance of the cascaded system is sensitive to laser and electron beam fluctuations.

In contrast, the EEHG technique uses a double modulator-chicane system to produce high harmonic bunching, and is capable of producing very high harmonics with relatively small energy modulations. In principle, this allows the production of soft $\mathrm{x}$ rays directly from UV seed lasers in a single stage. Furthermore, due to the highly nonlinear phase space manipulations inherent to the EEHG technique, the spectrum of an EEHG FEL is found to be highly insensitive to beam imperfections. These advantages have attracted a world-wide interest in EEHG as a way to produce fully coherent $x$ rays in seeded FELs. Recent initial efforts have demonstrated the 3rd and 4th harmonic in EEHG in 2010 [34,35], and the 7th harmonic was demonstrated in 2011 [36]. Here we report on the generation of highly coherent and stable vacuum ultraviolet (VUV) radiation at the 15th harmonic of an infrared seed laser by the EEHG technique using relatively small energy modulations and a realistic beam with considerable nonlinear energy structure. The experiment demonstrates the distinct advantages of EEHG in a new regime, where the high frequency up-conversion efficiency and insensitivity to electron beam phase space imperfections almost paradoxically result from a highly nonlinear recoherence effect. These results compel further work towards fully coherent $\mathrm{x}$ rays by EEHG in emerging seeded FELs, where enhanced stability and laser quality facilitate the precision probing of nature at ultrashort and ultrafast time scales.

\section{HIGH-GAIN HARMONIC GENERATION AND ECHO-ENABLED HARMONIC GENERATION}

The creation of a charge density modulation at suboptical wavelengths in an electron beam with lasers is analogous to the manipulation of the electron bunch length in a magnetic bunch compressor. The difference is that the energy chirp (correlation between a particle's energy and its longitudinal position) is imprinted by lasers rather than radio-frequency (rf) cavities. The process of longitudinal bunch compression, to the first order, can be described as a linear transformation where the bunch length is reduced while the energy spread (conservation of phase space area) and peak current (conservation of charge) are both increased. This is achieved by first accelerating the beam off-crest in $\mathrm{rf}$ cavities to establish a correlated energy chirp (e.g., with bunch head having a slightly lower energy than the bunch tail), and then sending the beam through a dispersive chicane. The particles with lower energy are bent more in the chicane and therefore traverse a longer path length than the higher energy particles that are bent less. As a result, the low energy electrons slip back longitudinally while the high energy electrons catch up, which leads to bunch compression.

Replacing the rf cavity with a laser allows one to create much finer structures in beam phase space without changing the overall bunch length. This is because the laser wavelength is typically much shorter than the electron beam duration, so the laser does not give beam a net energy chirp. As a result, after dispersion the overall bunch duration is kept constant. Instead, the time-varying laser field imprints a sinusoidal energy chirp (called an energy modulation) on the beam phase space which leads to varying local compression/decompression of the beam current on the scale of the laser wavelength after dispersion. The result is a density modulation in the current distribution of the beam, both at the laser frequency and at its harmonics.

For example, the phase space evolution in HGHG scheme is shown in Fig. 1. A laser is first used to interact with the beam in a modulator (a short undulator) to produce sinusoidal energy modulation in beam phase space [Fig. 1(b)]. After passing through a chicane, half of the particles that have the negative energy chirp [blue particles in Figs. 1(b) and 1(c)] are compressed, while the other half with the positive energy chirp [red particles in Figs. 1(b) and 1(c)] are decompressed. As a result of this transformation, the energy modulation is effectively converted into a density modulation [see Fig. 1(d) where the beam density consists of many spikes equally separated by the laser wavelength] that contains harmonic frequency components of the laser fundamental frequency.

Analysis shows that the width of the current spike normalized to the laser wavelength is approximately $1 / A$, where $A=\Delta E / \sigma_{E}$ is the ratio of energy modulation amplitude $\Delta E$ to the beam energy spread $\sigma_{E}$. Therefore, in 

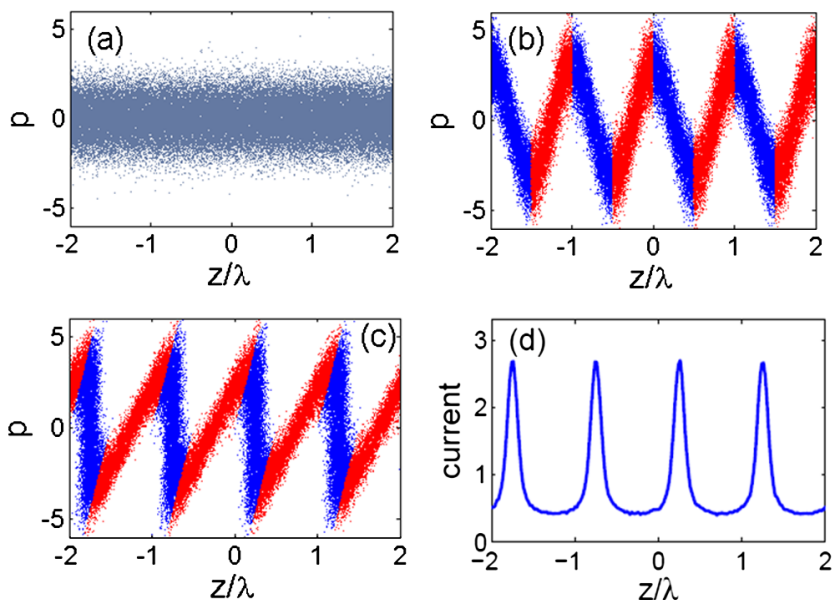

FIG. 1. Evolution of the longitudinal phase space in HGHG scheme: (a) before the modulator; (b) after the modulator; (c) after the chicane; (d) density distribution after the chicane normalized to the initial beam current. The horizontal axis is the beam longitudinal position normalized to the laser wavelength and the vertical axis is the particle's energy deviation with respect to the reference particle normalized to the rms slice energy spread of the beam. The energy modulation is 3 times larger than the beam energy spread.

general generation of the $h$ th harmonic requires the energy modulation to be approximately $h$ times larger than beam energy spread (note, with a non-Gaussian energy distribution, the harmonic number may be higher [37]), an undesired consequence for FEL applications where the increased energy spread may significantly reduce the FEL gain. Another undesired consequence for HGHG is that if the $h$ th harmonic is to be achieved, the peak current of the bump is also increased by about $h$ times [as can be seen in Fig. 1(d)], which may cause undesirable collective effects. Furthermore, the spectrum of the bunching is found to be sensitive to beam imperfections such as nonlinear beam energy chirps that may easily broaden the bunching spectrum by changing the separation of the current spikes [38,39].

These limitations can be overcome with the EEHG scheme in the double modulator-chicane system. Similar to the HGHG scheme, in EEHG the beam is also first energy modulated by a laser in the first modulator. But quite differently, the first chicane is chosen to have a large momentum compaction such that after passing through the strong chicane the density modulation is macroscopically smeared [Fig. 2(a)]. Simultaneously, complicated fine structures (e.g., "energy banding") are introduced into the phase space, which has been recently observed experimentally [40]. A second laser is then used to further modulate the beam energy in the second modulator [Fig. 2(b)] to imprint additional correlations in phase space. A second weaker chicane orients these correlations vertically in the energy space, which yields a charge density modulation at very high harmonic frequencies. As a result
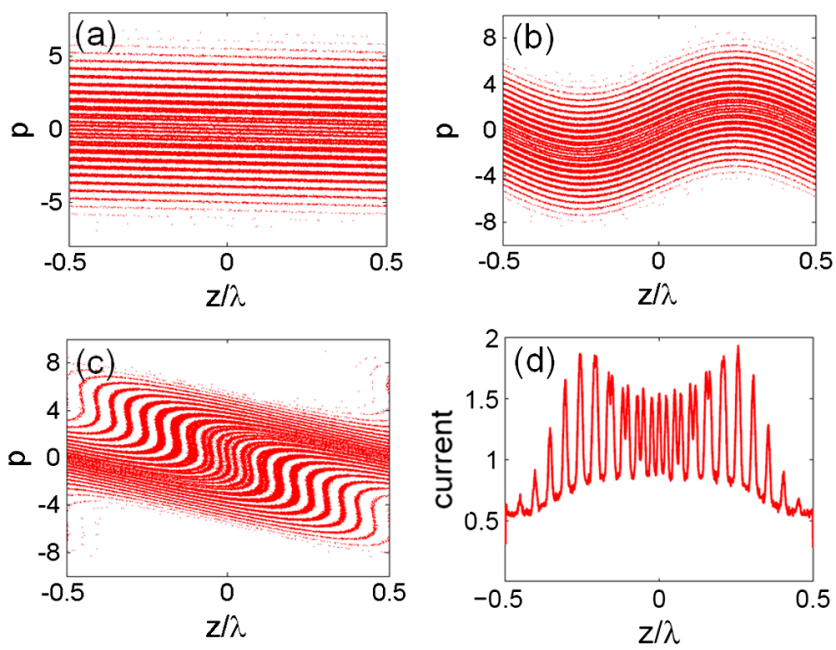

FIG. 2. Evolution of the longitudinal phase space in EEHG scheme: (a) after the first strong chicane; (b) after the second modulator; (c) after the second weak chicane; (d) density distribution after the second chicane.

of this nonlinear process, the harmonic bunching structure emerges as a recoherence effect after a short disappearance, like an echo [Figs. 2(c) and 2(d)].

The key advantage of the EEHG technique is that it can generate very high harmonics with harmonic number much larger than the ratio of energy modulation to energy spread, i.e., $n \gg \Delta E / \sigma_{E}$. This makes it possible to generate very high frequency bunching while simultaneously keeping the beam energy spread small, which allows the generation of soft $\mathrm{x}$ rays from a UV seed laser in a single stage. Another advantage of EEHG is that by splitting the single current bump into many bumps per wavelength, the peak current of each current bump can be significantly reduced, which effectively mitigates the potential collective effects related to the high peak current. For instance, as seen in Fig. 2(d), with the energy modulation 3 times larger than the beam energy spread, the peak current is only increased by a factor of 2 while the harmonic number is extended to $\sim 20$. Even higher harmonics can be produced by increasing the momentum compaction of the first chicane while keeping the energy modulation amplitudes essentially unchanged. Furthermore, due to the highly nonlinear phase space gymnastics that strongly decompress local regions of the beam, the spectrum of the bunching is made to almost immune to the phase space imperfections. This allows the generation of transform-limited highly coherent radiation in realistic conditions when the beam has considerable nonlinear chirps [41].

\section{EXPERIMENTAL RESULTS}

\section{A. Experimental setup}

Here we report on experimental studies of EEHG at a new harmonic regime $(h=15)$ in which highly coherent 


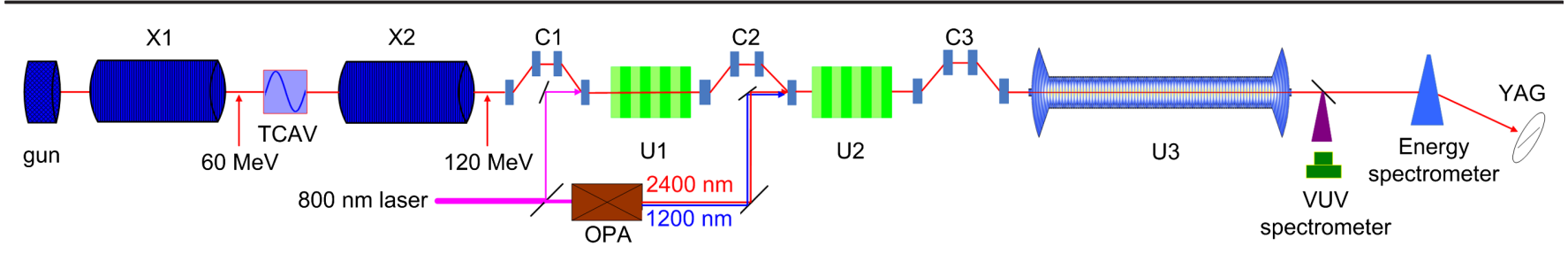

FIG. 3. Schematic of the EEHG experiment at SLAC. The beam is modulated by two lasers at 800 and $2400 \mathrm{~nm}$, and coherent VUV radiation at $160 \mathrm{~nm}$ as the 15 th harmonic of the second laser is produced in a microwave undulator (U3).

and stable VUV radiation is produced with the EEHG technique in realistic scenarios when the electron beam phase space has considerable linear and nonlinear energy chirp. The layout of our experiment performed at SLAC's Next Linear Collider Test Accelerator (NLCTA) using a $120 \mathrm{MeV}$ beam is schematically shown in Fig. 3. The electron beam is generated in a photocathode $\mathrm{rf}$ gun ( $r f$ frequency at $2.856 \mathrm{GHz}$ ) and is boosted to $60 \mathrm{MeV}$ in an X-band linac structure (X1, rf frequency at $11.424 \mathrm{GHz}$ ). The beam slice energy spread is increased in an rf transverse cavity (TCAV) and the beam energy is further increased to $120 \mathrm{MeV}$ with the second X-band linac structure (X2). The beam then enters the main EEHG beam line which consists of three chicanes $(\mathrm{C} 1, \mathrm{C} 2$ and $\mathrm{C} 3)$ and three undulators (U1, U2 and U3).

The chicane $\mathrm{C} 1$ is used to generate an orbit bump to inject the $800 \mathrm{~nm}$ laser $(\sim 1 \mathrm{ps}$ FWHM $)$ into the first undulator U1 (ten periods with a period length of $3.3 \mathrm{~cm}$ and $K$ value of 1.82) to produce energy modulation in beam phase space. After passing through $\mathrm{C} 2$, separated energy bands are produced in the beam longitudinal phase space. The beam then interacts with the $2400 \mathrm{~nm}$ laser $(\sim 1 \mathrm{ps}$ FWHM) in the second undulator U2 (ten periods with a period length of $5.5 \mathrm{~cm}$ and $K$ value of 2.76) where additional energy modulation is superimposed on the separated energy bands. After passing through C3, density modulation at shorter wavelengths is generated through the recoherence effect. Finally, the density modulated beam is sent through an X-band microwave undulator U3 (70 periods with a period of $1.39 \mathrm{~cm}$ and variable $K$ value [42]) to produce coherent VUV radiation that is measured with a high resolution VUV spectrometer.

Effective interaction between the laser and electron beam is achieved when electron and laser beam overlap both spatially and temporally in the modulators. The spatial overlap between the $800 \mathrm{~nm}$ laser and the electron beam is achieved by steering the laser to the same position as the beam on the screens (not shown in Fig. 3) upstream and downstream of the undulators. The temporal overlap is achieved with two steps. First, an optical transition radiation screen downstream of each undulator is used to reflect out the laser and undulator radiation which is detected by a fast photodiode. By referencing the signals to an external trigger, the laser and beam can be synchronized to within approximately $30 \mathrm{ps}$. More precise timing is then done by using a scanning delay stage and measuring the beam energy spread growth with the high-resolution energy spectrometer. The second laser at $2400 \mathrm{~nm}$, produced with an optical parametric amplifier (OPA) pumped with the $800 \mathrm{~nm}$ laser, is made to overlap with the beam in U2 with the help of the $1200 \mathrm{~nm}$ signal beam from the OPA. In the OPA [43], one photon is divided into two photons for which the sum energy equals to the energy of the pump photon. As a result, in our setup the idler at $2400 \mathrm{~nm}$ with polarization parallel to the wiggling direction in U2 is accompanied by the signal beam at $1200 \mathrm{~nm}$ which has the orthogonal polarization. Therefore, by steering the $1200 \mathrm{~nm}$ laser to the same position as the electron beam on the optical transition radiation screens (not shown in Fig. 3) upstream and downstream of $U 2$, the beam is made to overlap with the $2400 \mathrm{~nm}$ laser as well. After this procedure, a bandpass filter centered at $2400 \mathrm{~nm}$ is used in the experiment to ensure that the beam is only modulated by the $2400 \mathrm{~nm}$ laser in U2.

The energy modulation amplitudes are measured with an energy spectrometer with a dispersion of $1.5 \mathrm{~m}$ and a highresolution yttrium aluminum garnet screen. The beam slice energy spread is controlled with an 11-cell X-band transverse cavity (TCAV) which is an rf structure operating in the TM10 mode. The longitudinal electric field of a TCAV varies linearly with transverse distance and thus leads to an increase in beam slice energy spread by $\sigma_{E, T}=2 \pi e V \sigma_{x} / \lambda_{\mathrm{rf}}$, where $V$ is the voltage of the cavity, $\lambda_{\mathrm{rf}}$ is the wavelength of the rf field and $\sigma_{x}$ is the rms beam size in the cavity $[36,44]$.

\section{B. Highly coherent radiation from EEHG}

In EEHG, two lasers and two chicanes are used to manipulate the electron beam phase space to produce density modulation at the wave number $k_{E}=n k_{1}+m k_{2}$ with a bunching factor given as

$$
\begin{aligned}
b_{n, m}= & \mid e^{-\frac{1}{2}\left[n B_{1}+(\kappa m+n) B_{2}\right]^{2}} J_{m}\left[-(\kappa m+n) A_{2} B_{2}\right] \\
& \times J_{n}\left\{-A_{1}\left[n B_{1}+(\kappa m+n) B_{2}\right]\right\} \mid,
\end{aligned}
$$

where $n$ and $m$ are integers, $k_{1}$ and $k_{2}$ are the wave numbers of the first and second laser, $A_{1}=\Delta E_{1} / \sigma_{E}$ and $A_{2}=$ $\Delta E_{2} / \sigma_{E}$ are the energy modulation amplitude of the first and second laser normalized to the beam slice energy spread, $B_{1}=R_{56}^{(1)} k_{1} \sigma_{E} / E_{0}$ and $B_{2}=R_{56}^{(2)} k_{1} \sigma_{E} / E_{0}$ are the 
dimensionless momentum compactions of the first and second chicane, $E_{0}$ is the average beam energy and $\kappa=k_{2} / k_{1}$.

In our experiment the TCAV is first turned off such that beam slice energy spread is small (below $1 \mathrm{keV}$ from simulation) and both HGHG and EEHG signals at high harmonics can be produced. The momentum compaction of $\mathrm{C} 2$ and $\mathrm{C} 3$ are set at $R_{56}^{(1)}=4.8 \mathrm{~mm}$ and $R_{56}^{(2)}=1.0 \mathrm{~mm}$ to produce bunching at the 15 th harmonic of the second laser, corresponding to $n=-1$ and $m=18$ in Eq. (1). The laser energy modulations are first roughly set at $\Delta E_{1}=80 \mathrm{keV}$ and $\Delta E_{2}=65 \mathrm{keV}$ with the energy spectrometer following EEHG theory, and then finely adjusted with wave plates to maximize the EEHG signal. The optimized EEHG signal, produced by sending the density modulated beam $(5 \mathrm{pC}$ charge, 1 ps FWHM) through the microwave undulator, is measured with a VUV spectrometer (with a resolution of about $0.27 \mathrm{~nm}$ (FWHM) determined through measurement of the spectral width of the zeroth order radiation of the grating) and shown in Fig. 4(a). The intensity of the coherent VUV radiation at $160 \mathrm{~nm}$ is about 2 orders of magnitude higher than the incoherent undulator radiation. In addition to the 15th harmonic, moderate signals at the 14th and 16th harmonics with intensities 1 order of magnitude above incoherent radiation are also observed.

With the $800 \mathrm{~nm}$ laser off, the HGHG signal produced by the $2400 \mathrm{~nm}$ laser individually shows a double-peak spectrum with significantly reduced intensity [magenta solid line in Fig. 4(b)]. The optimized HGHG signal [red dashed line in Fig. 4(b)] is obtained by lowering the energy modulation amplitude $\Delta E_{2}$ by about $20 \%$. This is in good agreement with theories since for EEHG the bunching factor at the 15th harmonic is related to the Bessel function of the 18th order $(n=-1$ and $m=18)$ while that
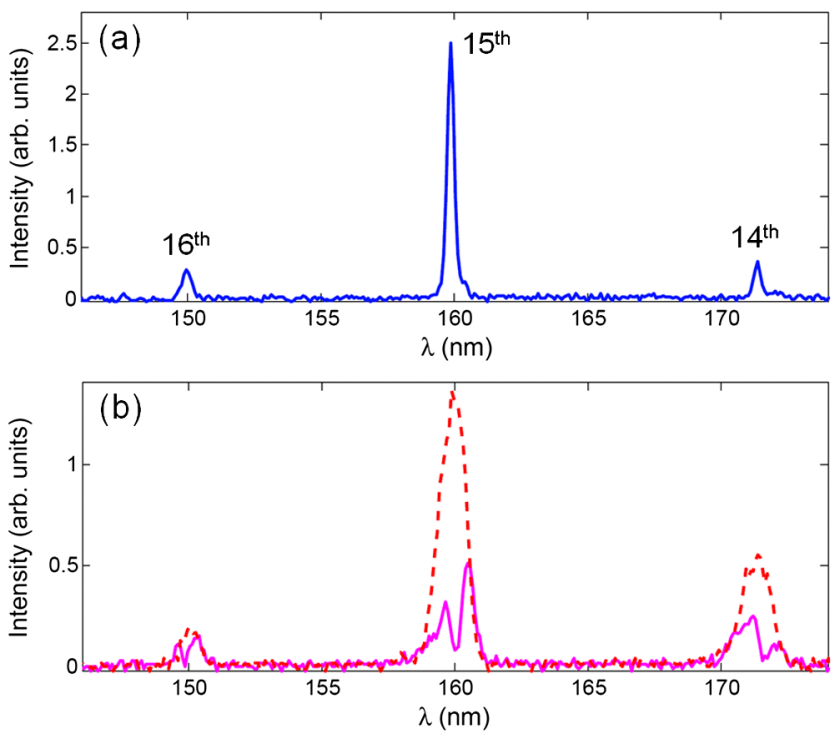

FIG. 4. Representative single-shot radiation spectrum for EEHG (a) and HGHG (b). for HGHG is related to the Bessel function of the 15th order ( $n=0$ and $m=15$ ) [25,26]. A comparison between the optimized EEHG signal and the optimized HGHG signal indicates that the EEHG signal has higher spectral brightness and smaller bandwidth.

To show the stability and repeatability of the measurements, ten consecutive radiation spectra of the optimized EEHG and HGHG signals obtained with the beam accelerated on-crest in the linac structures are shown in Fig. 5. The bandwidth of the optimized HGHG signal, averaged over 50 shots, is found to be about $1.02 \pm 0.21 \mathrm{~nm}$ (FWHM) while that for EEHG signal is only about $0.38 \pm$ $0.06 \mathrm{~nm}$ (FWHM). The averaged spectral brightness of EEHG is found to be about $60 \%$ higher than HGHG.

To understand the difference between the radiation spectra, we performed simulations using realistic electron beam and laser parameters. The beam center is assumed to temporally overlap with the center of the laser pulse, and the time-dependent beam current and laser energy modulation amplitudes are taken into account. The beam longitudinal phase space at the entrance to U1 used in the simulation is shown in Fig. 6(a). The electron beam is accelerated on-crest in the linac structure, and the nonlinear energy chirp from the varying rf phase along the bunch is included. The energy chirp of the beam together with the momentum compaction of $\mathrm{C} 3$ shifts the HGHG bunching wavelength to $\lambda=\lambda_{0} / C$, where $\lambda_{0}$ is the radiation wavelength when the beam has vanishing energy chirp, $C$ is the compression factor of the chicane [38]. Specifically, the bunching produced by the electrons in the bunch head (with a negative chirp corresponding to compression) is blueshifted while that produced by the electrons in the bunch tail (with a positive chirp corresponding to decompression) is redshifted. This nonlinear energy chirp leads to broadening of the HGHG spectrum, as shown with red dashed line in Fig. 6(b). In contrast, the EEHG bunching is much less sensitive to the beam nonlinear energy chirp, because of the strong shredding
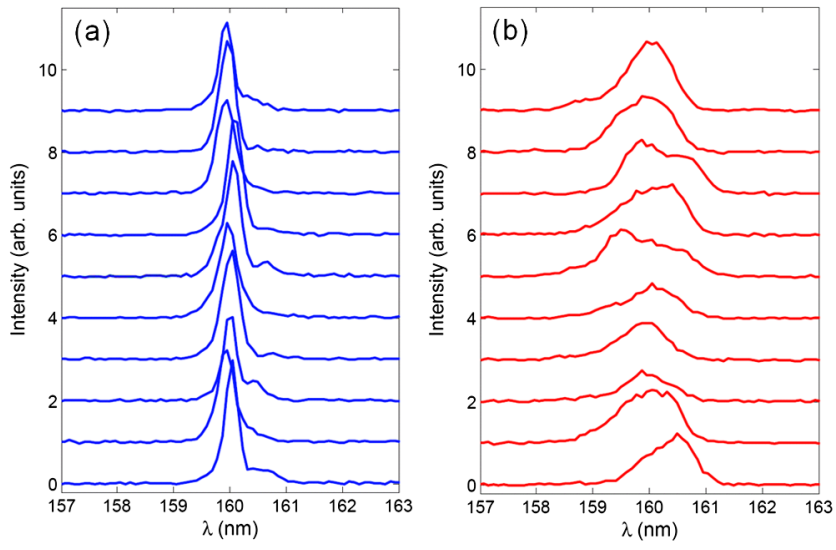

FIG. 5. Ten consecutive radiation spectra for EEHG (a) and HGHG (b) with an unchirped beam. 

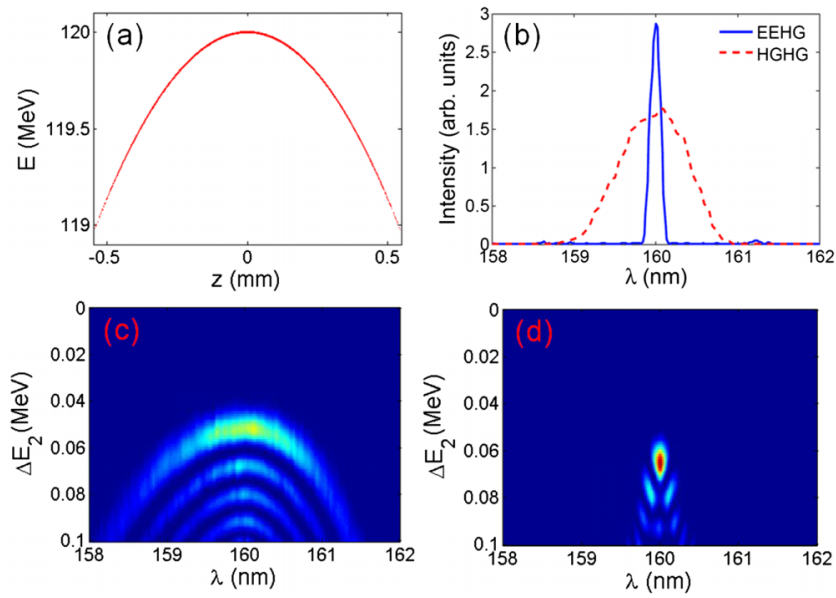

FIG. 6. Electron beam longitudinal phase space at the entrance to the EEHG beam line (a); simulated optimal EEHG and HGHG radiation spectra (b); HGHG spectra for various energy modulations (c); EEHG spectra for various energy modulations (d). (c) and (d) use the same scaling as Fig. 4.

of the phase space in the first chicane [41]. The simulated FWHM bandwidth of EEHG signal is found to be about $0.14 \mathrm{~nm}$ [blue solid line in Fig. 6(b)] and that for HGHG signal is about $1.00 \mathrm{~nm}$. This is in agreement with the experimental results, taking into account the resolution of the VUV spectrometer.

To understand the double-peaked spectrum of HGHG in Fig. 4(b), we simulated the bunching spectrum for various energy modulations in $\mathrm{U} 2$ taking into account the effect of finite laser pulse length. In the simulation, the HGHG spectral brightness is maximized when the peak energy modulation is $\Delta E_{2} \approx 52 \mathrm{keV}$ [Fig. 6(c)] and EEHG spectral brightness is maximized when $\Delta E_{2} \approx 65 \mathrm{keV}$ [Fig. 6(d)]. For HGHG with $\Delta E_{2} \approx 65 \mathrm{keV}$, the beam center is overbunched while the beam head and tail are optimally bunched. Note, the time-dependent bunching may be estimated with Eq. (1) by replacing $A_{1,2}$ with $A_{1,2}(t)=A_{1,2} e^{-t^{2} / 4 \sigma_{t}^{2}}$ to take into account the variation of the energy modulation along the bunch, where $\sigma_{t}$ is the rms laser pulse width. The time-dependent bunching together with the quadratic energy chirp leads to a dip in the center of the spectrum and double side peaks at longer and shorter wavelengths, in good agreement with the experimental results in Fig. 4(b). This effect is consistent with earlier observations and has been used to produce two-color $\mathrm{x}$-ray pulses in seeded FELs [45-47]. Further increasing the energy modulation amplitude will result in more peaks in the HGHG spectrum, whereas lowering it by about $20 \%$ leads to the optimal HGHG signal, as shown in Fig. 6(b) and observed in Fig. 4(b) (red dashed line). For EEHG, the weak dependence of bunching frequency on beam energy chirp enables a relatively narrow spectrum over a large range of energy modulation amplitudes.
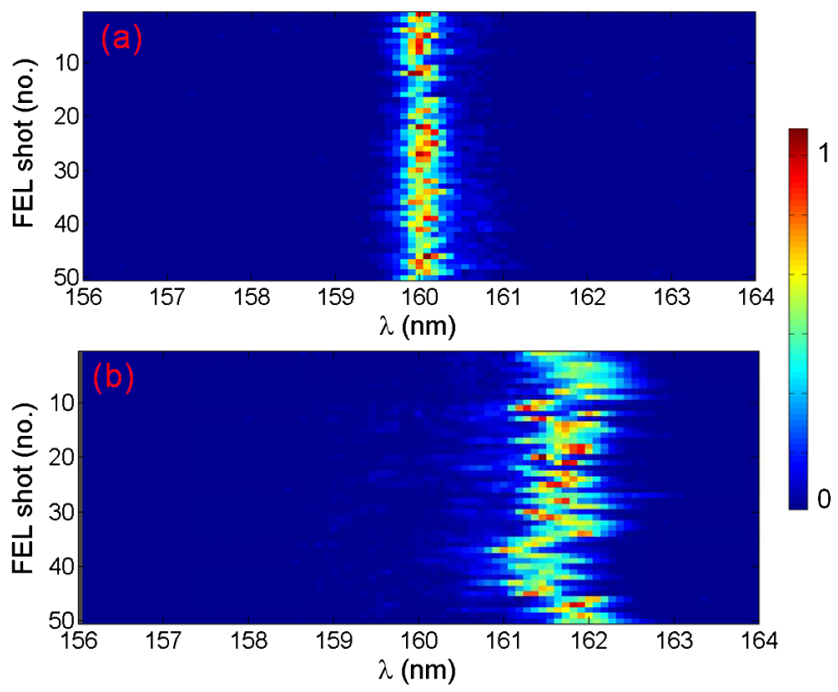

FIG. 7. Fifty consecutive radiation spectra for EEHG (a) and HGHG (b) with a chirped beam. Note, the central wavelength of HGHG signal is shifted by the linear chirp and the bandwidth of the HGHG signal is increased by the nonlinear chirp, while those for EEHG are essentially unaffected.

\section{Highly stable radiation from EEHG}

The different sensitivity of EEHG and HGHG on beam phase space imperfections is clearly illustrated with a chirped electron beam. In this separate experiment, the beam is accelerated at an off-crest phase (about 2.5 degrees from on-crest phase) to imprint a positive chirp in beam longitudinal phase space. In this case the EEHG and HGHG radiation spectra for 50 consecutive shots are shown in Fig. 7.

The rf phase jitter leads to fluctuations in beam energy chirp that together with the timing jitter between the laser and electron beam result in variation of the central wavelength in HGHG radiation, as shown in Fig. 7(b). Also the spectrum is broadened by the nonlinear beam energy chirp. In contrast, the central wavelength of EEHG signals stays essentially at the same value [Fig. 7(a)] and the spectrum bandwidth is much narrower. This may have profound implications for future seeded FELs where the wakefields of the acceleration structure and microbunching instabilities may lead to considerable high-order correlations in beam longitudinal phase space that may cause noticeable degradations to the radiation temporal coherence.

\section{High frequency up-conversion efficiency from EEHG}

In addition to confirming EEHG's relative immunity to beam imperfections, we increased the beam slice energy spread with the TCAV in a separate experiment to demonstrate the high frequency up-conversion efficiency of EEHG. With the voltage of the TCAV set at $450 \mathrm{kV}$ determined by measuring the maximal deflection angle of 


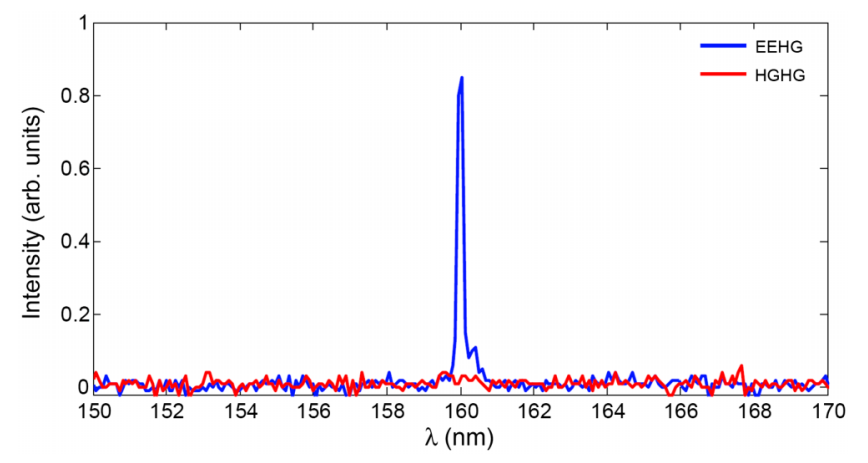

FIG. 8. EEHG and HGHG signals with the beam slice energy spread increased by a TCAV.

the beam, considerable EEHG signal at the 15th harmonic is still generated while the HGHG signal is completely suppressed, as shown in Fig. 8. The beam size at the entrance to the TCAV is measured to be about $100 \mu \mathrm{m}$, and the resulting beam slice energy spread is estimated to be about $10 \mathrm{keV}$. It should be pointed out that it is the slice beam size rather than the projected beam size that determines the slice energy spread growth. So the value estimated from the measured projected beam size should be considered as the upper limit of the true slice beam energy spread. Furthermore, while bunching at still higher harmonics (theory predicts considerable bunching at $h=30$ and $h=45$ ) may be produced in the beam, radiation at such short wavelengths cannot be generated with our low beam energy. In the future we plan to install another linac structure following C3 to study the frequency up-conversion efficiency of EEHG in the ultrahigh harmonic regime.

\section{DISCUSSIONS}

We have presented measurements of highly coherent and stable VUV radiation produced through the EEHG technique when the beam has a considerable nonlinear chirp. The capability to produce the 15 th harmonic with an energy modulation amplitude only about 6 times larger than the beam energy spread has also been demonstrated. These results highlight several potential advantages of EEHG in realistic conditions, namely, that the high frequency upconversion efficiency allows one to reach soft $\mathrm{x}$ rays in seeded FELs in a single stage, and that the insensitivity to electron beam phase space imperfections allows the production of narrow bandwidths with a stable central wavelength in spite of strong linear and nonlinear correlations in the phase space. Our results should forward the development of future seeded x-ray FELs that aim to produce laserlike $\mathrm{x}$ rays which may enable many new areas of sciences.

\section{ACKNOWLEDGMENTS}

We thank R. Coffee and G. Stupakov for useful discussions, and M. Shumail, S. Tantawi, and F. Wang for help in operation of the rf transverse cavity and $\mathrm{rf}$ undulator. This work was supported by the U.S. DOE Office of Basic Energy Sciences using the NLCTA facility which is partly supported by U.S. DOE Office of High Energy Physics under Contract No. DE-AC02-76SF00515. One of the authors (D.X.) would like to thank the support from DOE Early Career Award from 2012 to 2014.

[1] R. Neutze, R. Wouts, D. van der Spoel, E. Weckert, and J. Hajdu, Nature (London) 406, 752 (2000).

[2] H. Chapman et al., Nature (London) 470, 73 (2011).

[3] M. Marvin Seibert et al., Nature (London) 470, 78 (2011).

[4] N. D. Loh et al., Nature (London) 486, 513 (2012).

[5] C. Weninger, M. Purvis, D. Ryan, R. London, J. Bozek, C. Bostedt, A. Graf, G. Brown, J. Rocca, and N. Rohringer, Phys. Rev. Lett. 111, 233902 (2013).

[6] S. de Jong et al., Nat. Mater. 12, 882 (2013).

[7] T. Glover et al., Nature (London) 488, 603 (2012).

[8] W. Ackermann et al., Nat. Photonics 1, 336 (2007).

[9] P. Emma et al., Nat. Photonics 4, 641 (2010).

[10] T. Ishikawa et al., Nat. Photonics 6, 540 (2012).

[11] A. Kondratenko and E. Saldin, Part. Accel. 10, 207 (1980).

[12] R. Bonifacio, C. Pellegrini, and L.M. Narducci, Opt. Commun. 50, 373 (1984).

[13] J. Feldhaus, E. L. Saldin, J. R. Schneider, E. A. Schneidmiller, and M. V. Yurkov, Opt. Commun. 140, 341 (1997).

[14] G. Geloni, V. Kocharyan, and E. Saldin, J. Mod. Opt. 58, 1391 (2011).

[15] J. Amann et al., Nat. Photonics 6, 693 (2012).

[16] T. Popmintchev, M.-C. Chen, P. Arpin, M. M. Murnane, and H. C. Kapteyn, Nat. Photonics 4, 822 (2010).

[17] G. Lambert et al., Nat. Phys. 4, 296 (2008).

[18] T. Togashi et al., Opt. Express 19, 317 (2011).

[19] S. Ackermann et al., Phys. Rev. Lett. 111, 114801 (2013).

[20] L.-H. Yu, Phys. Rev. A 44, 5178 (1991).

[21] B. McNeil, G. Robb, and M. Poole, in Proceedings of the 21 st Particle Accelerator Conference, Knoxville, TN, 2005 (IEEE, Piscataway, NJ, 2005), p. 1718.

[22] E. Allaria and G. De Ninno, Phys. Rev. Lett. 99, 014801 (2007).

[23] Q. Jia, Appl. Phys. Lett. 93, 141102 (2008).

[24] H. Deng and C. Feng, Phys. Rev. Lett. 111, 084801 (2013).

[25] G. Stupakov, Phys. Rev. Lett. 102, 074801 (2009).

[26] D. Xiang and G. Stupakov, Phys. Rev. ST Accel. Beams 12, 030702 (2009).

[27] D. Xiang and G. Stupakov, New J. Phys. 13, 093028 (2011).

[28] E. Hemsing and D. Xiang, Phys. Rev. ST Accel. Beams 16, 010706 (2013).

[29] E. Hemsing, G. Stupakov, D. Xiang, and A. Zholents, Rev. Mod. Phys. 86, 897 (2014).

[30] L.-H. Yu et al., Science 289, 932 (2000).

[31] E. Allaria et al., Nat. Photonics 6, 699 (2012).

[32] J. Wu and L. H. Yu, Nucl. Instrum. Methods Phys. Res., Sect. A 475, 104 (2001).

[33] E. Allaria et al., Nat. Photonics 7, 913 (2013).

[34] D. Xiang et al., Phys. Rev. Lett. 105, 114801 (2010). 
[35] Z. Zhao et al., Nat. Photonics 6, 360 (2012).

[36] D. Xiang et al., Phys. Rev. Lett. 108, 024802 (2012).

[37] E. Ferrari, E. Allaria, W. Fawley, L. Giannessi, Z. Huang, G. Penco, and S. Spampinati, Phys. Rev. Lett. 112, 114802 (2014).

[38] T. Shaftan and L.-H. Yu, Phys. Rev. E 71, 046501 (2005).

[39] A. Marinelli, C. Pellegini, L. Giannessi, and S. Reiche, Phys. Rev. ST Accel. Beams 13, 070701 (2010).

[40] E. Hemsing, D. Xiang, M. Dunning, S. Weathersby, C. Hast, and T. Raubenheimer, Phys. Rev. ST Accel. Beams 17, 010703 (2014).

[41] Z. Huang et al., in Proceedings of the 31st International Free Electron Laser Conference (FEL 09), Liverpool, UK
(STFC Daresbury Laboratory, Warrington, 2009), p. 127.

[42] S. Tantawi, M. Shumail, J. Neilson, G. Bowden, C. Chang, E. Hemsing, and M. Dunning, Phys. Rev. Lett. 112, 164802 (2014).

[43] G. Cerullo and S. De Silverstri, Rev. Sci. Instrum. 74, 1 (2003).

[44] C. Behrens, Z. Huang, and D. Xiang, Phys. Rev. ST Accel. Beams 15, 022802 (2012).

[45] D. Xiang et al., Phys. Rev. ST Accel. Beams 14, 112801 (2011).

[46] G. De Ninno, B. Mahieu, E. Allaria, L. Giannessi, and S. Spampinati, Phys. Rev. Lett. 110, 064801 (2013).

[47] E. Allaria et al., Nat. Commun. 4, 2476 (2013). 